\title{
Study of Calcium Distribution in Alzheimer's Disease Using Finite Element Technique
}

\author{
Devanshi D. Dave*, Brajesh Kumar Jha \\ Dept. of Mathematics, School of Technology, PDPU, Raisan, Gandhinagar-382007, Gujarat, India. \\ * Corresponding author. Email: ddave1822@gmail.com \\ Manuscript submitted February 11, 2018; accepted March 5, 2018. \\ doi: 10.17706/ijbbb.2019.9.1.35-41
}

\begin{abstract}
Alzheimer's disease is the most common neurodegenerative disorder that is prevailing worldwide. Out of many reasons that may have their roles in prevailing this dementia, higher level of calcium concentration is one of them. There are various parameters that have their hands in maintaining the calcium level of the cytosol. Here we have taken into consideration the buffers and voltage gated calcium channel (VGCC) and used a two dimensional mathematical model to get the desired results. Finite element method (FEM) is employed to carry out the computations. Exogenous buffers like EGTA and BAPTA, which lower down the calcium concentration, have been used in addition to VGCC, which increases the calcium flux at the cytosolic level, to study the impact of calcium in Alzheimer's disease. The obtained results show that there is increase in calcium concentration level of Alzheimer's affected cell which render toxicity to the cell followed by cell death in AD.
\end{abstract}

Key words: Alzheimer's disease, buffers, calcium, FEM, VGCC.

\section{Introduction}

Mathematical modeling and simulation of biological systems is done on a large scale in present era. The amalgamation of various techniques helps in delineating various physiological phenomena taking place. Human body is divided basically into central nervous system and peripheral nervous system. Brain and spinal cords comes under central nervous system [1]. Out of all, human brain is the most complex structure and hence the mathematical modeling of it is the favorite task of the researchers. Brain on the whole works as a syncytium due to millions of cells which are connected to billions of other cells. Neurons are known as the building blocks of central nervous system, whereas astrocytes, oligodendrocytes, microglia are supporting cell, which helps in maintaining the brain functions and physiological processes [1], [2]. Besides various cells, there are numerous ions inside the brain that plays important role. Calcium ion being a second messenger of the brain plays an important role in maintaining the physiological functions [3]. Calcium has its hand in functioning of brain and several other functions like synaptogenesis, gene expression, cell differentiation, proliferation, etc. [4]. Due to its widespread role of calcium in brain the slightest of the change in it would result in major changes in physical as well as physiological changes.

Various studies suggest that the changes in the calcium level may result in several neurodegenerative diseases. Alzheimer's disease, Parkinson's disease, Epilepsy, etc. are some of the neuronal disorder which may take place due to alterations in cytosolic calcium concentration level [5]-[8]. Out of all, Alzheimer's disease is the most common form of dementia which is prevailing worldwide. Being sporadic in nature, 
there is no perfect known cause of Alzheimer's to occur [9]. Amyloid beta plaques and neurofibrillary tangles are the two hallmark causes of Alzheimer's whereas, declination in memory power, loss of thinking capacity and linguistic skills, gait disturbance etc. are the physical symptoms of Alzheimer's disease [6], [10]-[12]. Research shows that the high level of calcium concentration level results in toxicity of the cell [13]. This toxicity is affected directly linked to the cell fate. The cell calcium is maintained by calcium binding buffers, certain calcium channels and exchanges and some entities like endoplasmic reticulum and mitochondria and the slightest of the change in any of the parameters would generate a plethora of dysfunctions and hence the neurodegenerativity [12]. Thus study of calcium diffusion is an important aspect to look upon. Here we have considered buffers and VGCC to study the calcium concentration distribution. Buffers, on one hand decreases the calcium level by binding it with calcium whereas on the other hand, VGCC increases the cytosolic calcium through the channel fluxes. Thus, the combination of both the considered parameters maintains the cell calcium and hence the alterations in it would lead to neurodegenerativity. The research done so far suggests that the decrease in calcium binding buffers and alterations in the voltage gated calcium channels may be the some of the cause of neurodegenerativity in Alzheimer's disease [14],[15]. Literature survey suggests that several authors have mathematically studied calcium diffusion in different cells in presence of buffers and VGCC [16]-[18]. They have considered the regular shape of the cell and employed finite element method to get the results. Keeping in mind the above physiological facts and literature survey, we have attempted to mathematically model the calcium diffusion taking place in presence of buffers and VGCC in approximated typical structure of neuron cell. Over and above the irregularity of the cell shape, the relation between the alterations in calcium and Alzheimer's disease is explained. The mathematical formulation, geometrical description, result and discussion are given in the next sections.

\section{Mathematical Formulation}

The mathematical formulation of the calcium buffering can be stated as [19], [20]

$$
\left[C a^{2+}\right]+[B] \Leftrightarrow[C a B]
$$

Here $[\mathrm{Ca} 2+]$ is the calcium concentration and $[\mathrm{B}]$ is the buffer concentration, whereas $[\mathrm{CaB}]$ is the calcium bound buffer concentration.

Using Fickian law, the resulting differential equations can be sated as [20], [21]

$$
\begin{gathered}
\frac{\partial\left[\mathrm{Ca}^{2+}\right]}{\partial t}=D_{C a}\left(\frac{\partial^{2}\left[\mathrm{Ca}^{2+}\right]}{\partial x^{2}}+\frac{\partial^{2}\left[\mathrm{Ca}^{2+}\right]}{\partial y^{2}}\right)+\sum_{j} R_{j} \\
\frac{\partial\left[B_{j}\right]}{\partial t}=D_{B_{j}}\left(\frac{\partial^{2}\left[B_{j}\right]}{\partial x^{2}}+\frac{\partial^{2}\left[B_{j}\right]}{\partial y^{2}}\right)+R_{j} \\
\frac{\partial\left[C a B_{j}\right]}{\partial t}=D_{C a B_{j}}\left(\frac{\partial^{2}\left[C a B_{j}\right]}{\partial x^{2}}+\frac{\partial^{2}\left[C a B_{j}\right]}{\partial y^{2}}\right)-R_{j}
\end{gathered}
$$

where

$$
R_{j}=-k_{j}^{+}\left[B_{j}\right]\left[\mathrm{Ca}^{2+}\right]+k_{j}^{-}\left[\mathrm{CaB}{ }_{j}\right]
$$

The symbol of $D_{C a}, D_{B_{j}}, D_{C a B_{j}}$ stands for diffusion coefficients for calcium, buffer and calcium bound 
buffers respectively. $k_{j}^{+}, k_{j}^{-}$are the association and the dissociation rates for buffer ' $\mathrm{j}$ ' respectively.

The formulation of VGCC can be done mathematically using the Goldman-Hodgkin-Katz (GHK) current equation as [22]

$$
I_{C a}=P_{C a} z_{C a}^{2} \frac{F^{2} V_{m}}{R T} \frac{\left[C a^{2+}\right]_{i}-\left[C a^{2+}\right]_{o} \exp \left(-z_{C a} \frac{F V_{m}}{R T}\right)}{1-\exp \left(-z_{C a} \frac{F V_{m}}{R T}\right)}
$$

Here $\left[\mathrm{Ca}^{2+}\right]_{i}$ is the intracellular concentration of calcium and $\left[\mathrm{Ca}^{2+}\right]_{o}$ is the extracellular calcium concentration. $P_{C a}$ is the permeability of calcium ion. ${ }^{z_{C a}}$ is the valency of calcium ion, $\mathrm{F}$ is Faraday's constant. $V_{m}$ is membrane potential. R is real gas constant. T is the absolute temperature.

To convert equation (6) into moles/second [16]

$$
\sigma_{C a}=\frac{-I_{C a}}{z_{C a} F V_{\text {nervecells }}}
$$

Using the mathematical formulation of calcium buffering and VGCC as internal processes, the computational model is stated as

$$
\frac{\partial c}{\partial t}=D_{x} \frac{\partial^{2} c}{\partial x^{2}}+D_{y} \frac{\partial^{2} c}{\partial y^{2}}-{ }_{m}^{k}\left[B_{\infty}\left(c-c_{\infty}\right)+\sigma_{V G C}\right.
$$

The boundary conditions according to physiology of the cell is [17]

$$
-D_{C a} \frac{\partial u}{\partial x}=\sigma_{C a}
$$

Physiologically, the background concentration of $\left[\mathrm{Ca}^{2+}\right]$ is considered $0.1 \mu \mathrm{M}$ [17]. Thus, another condition taken into consideration is

$$
\left[\mathrm{Ca}^{2+}\right]=0.1 \mu M
$$

The geometrical description of the approximated cell structure is shown in next section followed by results.

\section{Geometrical Description}
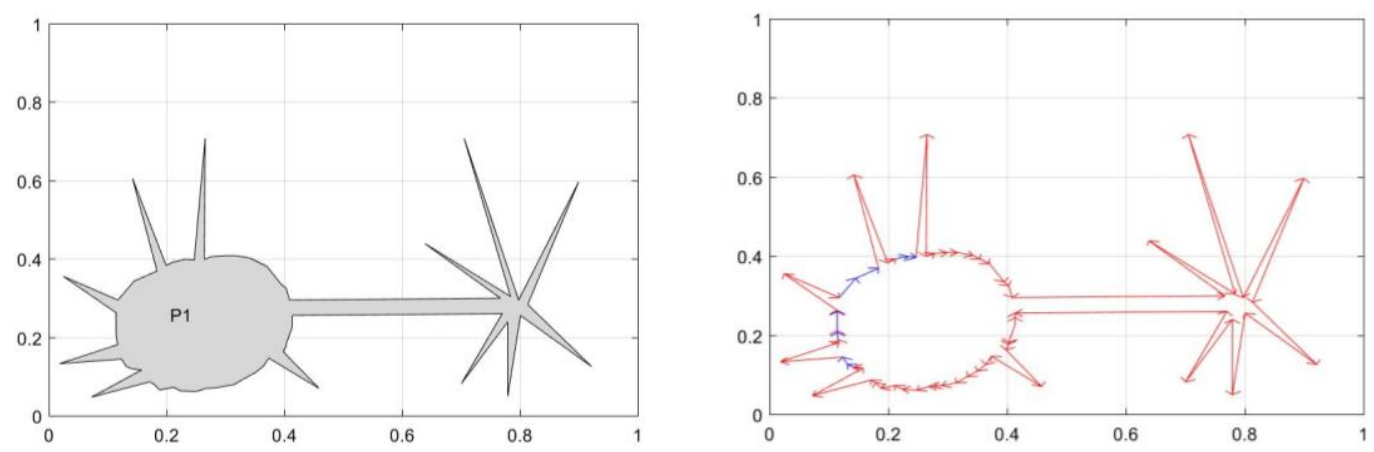

Fig. 1. Approximated geometry of the cell and boundary conditions incorporated on the cell. 
The approximated cell structure of a typical neuron cell along with the boundary conditions which are incorporated are shown in Fig. 1. These boundary conditions are taken in such a way that it is in harmony with the physiological condition of the cells. Here multi boundaries are taken into consideration which is due to the physiological calcium flux taking place through the borders in cell.

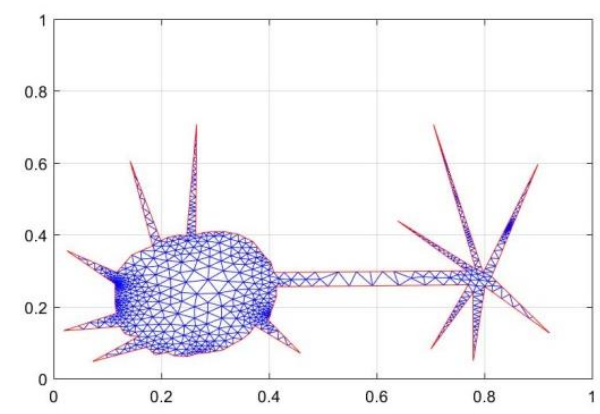

Fig. 2. Discretized domain of the cell in triangular elements.

Further, Fig. 2 shows the discretized cell, i.e. entire domain is discretized into small elements. Entire domain of the cell is divided into 1311 triangular elements having 833 nodal points. Triangular elements are the basic elements used for approximating the solution on the entire domain.

\section{Results and Discussion}
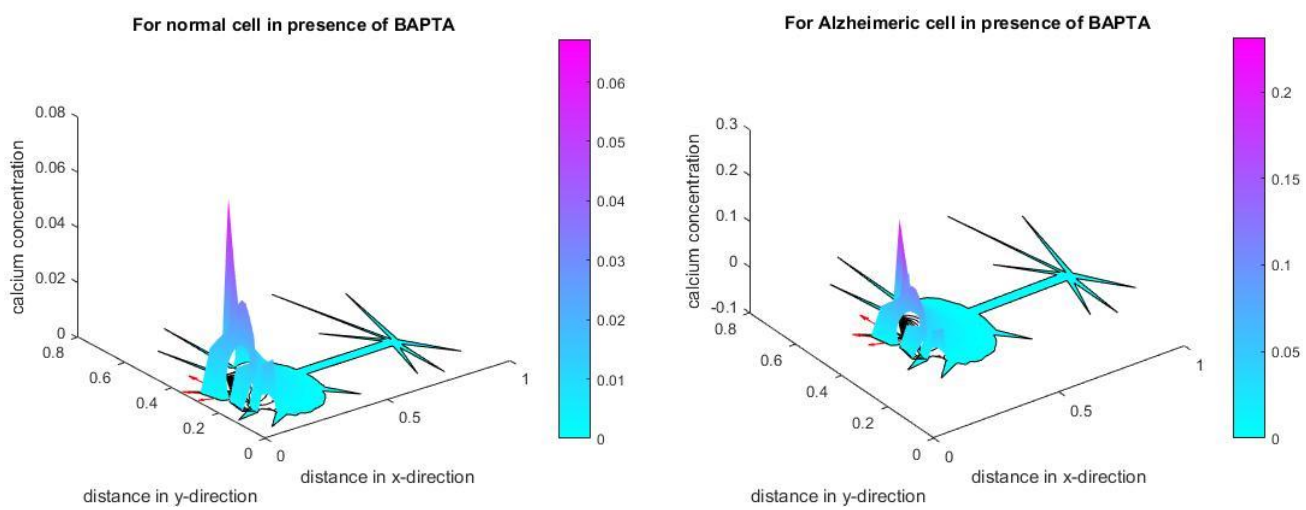

Fig. 3. Calcium concentration distribution in presence of BAPTA and VGCC for normal and Alzheimeric cells.

Fig. 3 shows the calcium concentration (diffusion coefficient 200-300 $\mu \mathrm{m}^{2} \mathrm{~s}^{-1}$ ) distribution in presence of BAPTA having association rate of $600 \mu \mathrm{m}^{-1} \mathrm{~s}^{-1}$. BAPTA is known as the exogenous buffer. The graphs are shown for the normal and Alzheimeric condition of the cell. Physiologically it has been found that the lower levels of the calcium buffers results into the higher amount of calcium in the cytosol [9]. Also, literature shows that there is disturbance in the voltage gated calcium channels which also results in alterations in calcium concentration level [12]. Here both the parameters are taken into consideration and their impact is shown in Fig. 3. Fig. 3a shows the normal condition of the cell whereas the Fig. $3 \mathrm{~b}$ having higher amount of calcium shows the Alzheimeric condition of the cell. The level of calcium in normal cell is round about 0.08 whereas it is 0.3 for Alzheimer's affected cell and then attains the background concentration of $0.1 \mu M$. The higher amount of the cell disturbs the normal functioning of the cell followed by cell loss and cell death cascades. Due to this, the new formation of memory won't take place and the symptoms of the Alzheimer's disease starts taking place. This higher amount of calcium renders toxicity to the cell and hence the cells 
become vulnerable to Alzheimer's disease.
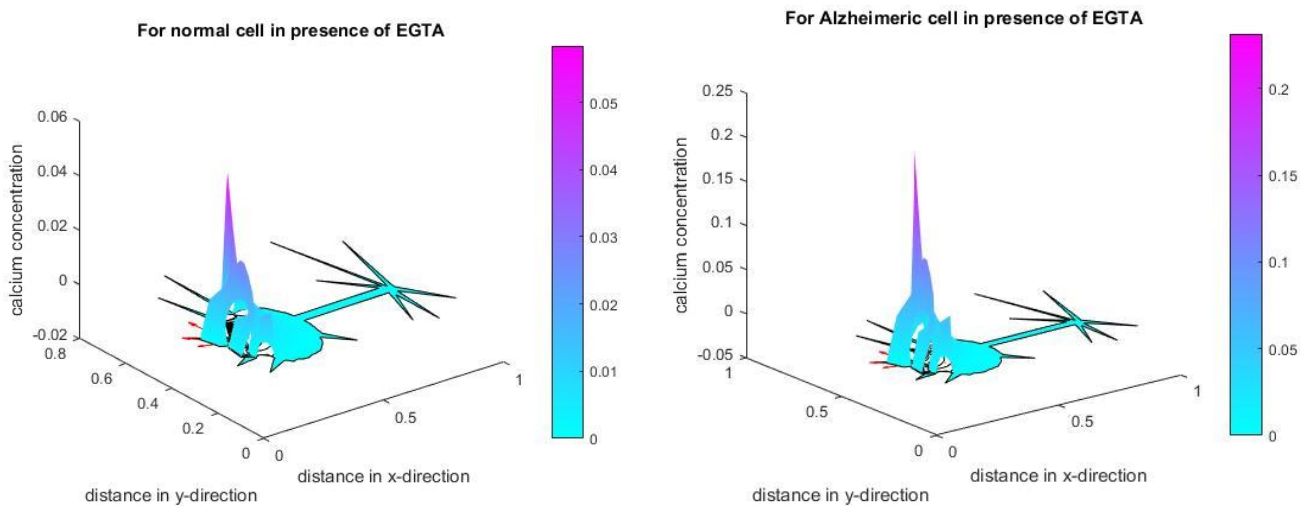

Fig. 4. Calcium concentration distribution in presence of EGTA and VGCC for normal and Alzheimeric cells.

Fig. 4 shows the calcium concentration distribution in presence of EGTA having association rate of 1.6 $\mu m^{-1} s^{-1}$ and VGCC. Here also, in Alzheimeric cell buffer loses its amount and binding affinity and the activity of calcium channel is altered. The changes due to these alterations can clearly be seen from Fig. 4a and Fig. 4b. The calcium concentration for normal cell is round about 0.06 and for Alzheimeric cell is 0.25. The significant impact of the both the parameters on the cytosolic calcium concentration can be seen from both the figures. Hence, it is observed that the slightest of the modifications in the buffer amount, buffer affinity and the voltage gated calcium channel may result into neurodegenerativity of Alzheimer's disease. There is difference in calcium hike for both of the buffers due to the difference in the binding affinity of EGTA and BAPTA.

\section{Conclusion}

In the present paper, an attempt has been made to study the impact of calcium concentration in presence of buffers and VGCC for normal and Alzheimeric cells. For this, a two dimensional mathematical model is used having initial and boundary conditions matching the physiology of the brain. Further, finite element method is used to get the results. The fundamental procedure of the finite element method is followed, i.e. the domain is drawn and after appropriate boundary conditions, the domain is discretized and the approximate solution is obtained. Two different buffers namely, BAPTA and EGTA are used in presence of VGCC. The significant and noteworthy change in the normal and Alzheimeric cell is shown for cytosolic calcium concentration level. The fact that the increase in calcium concentration leads to the neurodegenerativity of Alzheimer's disease can be verified from Fig. 3 and Fig. 4. Here the results obtained are totally computational and it needs to be verified by the biologists so that they can be used it in future for unrevealing the hidden part of Alzheimer's. Further such models can be developed to get better insights about the physiology behind the neurodegenerative diseases and its connection with diverse parameters like sodium calcium exchanger, voltage gated calcium channels, endoplasmic reticulum, mitochondria, etc. taking place in all the nerve cells.

\section{References}

[1] Squire, L., et al. (2008). Fundamental Neuroscience. Elsevier.

[2] Verkhratsky, A., \& Butt, A. (2007). Glial Neurobiology: A Textbook. John Wiley \& Sons, Ltd.

[3] Clapham, D. E. (2007). Calcium signaling. Cell, 131, 1047-1058.

[4] Augustine, G. J., Santamaria, F., \& Tanaka, K. (2003). Local calcium signaling in neurons. Neuron, 40, 
331-346.

[5] Cano-abad, M. F., et al. (2011). New insights on culture and calcium signalling in neurons and astrocytes from epileptic patients. Int. J. Dev. Neurosci., 29, 121-129.

[6] Magi, S., et al. (2016). Intracellular calcium dysregulation: Implications for Alzheimer' s disease. Biomed Res. Int., 2016, 1-14.

[7] Bezprozvanny, I. (2011). Calcium signalling and neurodegenerative diseases. Trends Mol Med., 15(3), 89-100.

[8] Berridge, M. J. (2012). Dysregulation of neural calcium signaling in Alzheimer disease, bipolar disorder and schizophrenia. Prion, 6(5), 1-12.

[9] Turkington, C., \& Mitchell, D. (2010). The Encyclopedia of Alzheimer's Disease. Facts on File: An Imprint of Infobase Publishing.

[10] Laferla, F. M. (2002). Calcium dyshomeostasis and intracellular signalling in Alzheimer's disease. Nat Rev Neurosci, 3, 862-872.

[11] Mattson, M. P., \& Chan, S. L. (2003). Neuronal and glial calcium signaling in Alzheimer's disease. Cell Calcium, 34, 385-397.

[12] Brawek, B., \& Garaschuk, O. (2014). Network-wide dysregulation of calcium homeostasis in Alzheimer's disease. Cell Tissue Res.

[13] Green, K. N., \& Laferla, F. M. (2008). Linking calcium to AB and Alzheimer's disease. Neuron, 59, 190-194.

[14] Riascos, D., et al. (2011). Age-related loss of calcium buffering and selective neuronal vulnerability in Alzheimer's disease. Acta Neuropathol, 122, 565-576.

[15] Nikoletopoulou, V., \& Tavernarakis, N. (2012). Calcium homeostasis in aging neurons. Front. Genet., 3, $1-17$.

[16] Dave, D. D., \& Jha, B. K. (2018). Modeling the alterations in calcium homeostasis in the presence of protein and VGCC for Alzheimeric cell. Advances in Intelligent Systems and Computing, 181-189.

[17] Jha, B. K., et al. (2013). Two-dimensional finite element model to study calcium distribution in astrocytes in presence of VGCC and excess buffer. Int. J. Model. Simulation, Sci. Comput., 4(2).

[18] Naik, P. A., \& Pardasani, K. R. (2016). Finite element model to study calcium distribution in oocytes involving voltage gated Ca 2 + channel, ryanodine receptor and buffers. Alexandria J. Med., 52(1), 43-49.

[19] Smith, G. D. (1996). Analytical steady-state solution to the rapid buffering approximation near an open Ca2+ channel. Biophys. J., 71, 3064-3072.

[20] Smith, G. D., et al. (2001). Asymptotic analysis of buffered calcium diffusion near a point source. SIAM J. APPL. MATH., 61(5), 1816-1838.

[21] Crank, J. (1975). The Mathematics of Diffusion. Clarendon Press Oxford.

[22] Keener, J., \&Sneyd, J. (2009). Mathematical Physiology. Springer US.

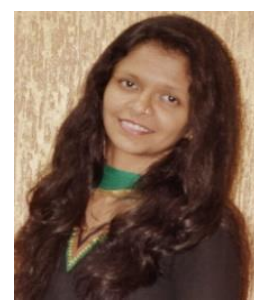

Devanshi D. Dave did her bachelor of science in mathematics from C. U. Shah Science College, Ahmedabad, Gujarat, India, in the year 2012. Then she joined in School of Sciences, Gujarat University, Gujarat, India for the master of science and completed it with mathematics as a specialized subject in the year 2014. Later on, in the year 2015 she joined at Pandit Deendayal Petroleum University, Gandhinagar, India as a research scholar and is currently pursuing PhD.

She is involved in research in the field of computational neuroscience. She has published 5 research papers and presented 10 research papers in various national/ international conferences and seminars. She is a life member of societies like GAMS and FATER. 
Brajesh Kumar Jha did his bachelor of mathematics in 2005 from Ram Krishna College, Madhubani, Bihar, India and the master of mathematics in 2007 from School of Mathematics and Allied Sciences, SOMAAS, Jiwaji University, Gwalior, M.P., India. He has completed his Ph.D. in applied mathematics in 2013 from S. V. National Institute of Technology, Surat, India. His areas of interest are computational neuroscience, biomathematics, finite element method, etc. Currently he is working as an Assistant Professor of Mathematics at Pandit Deendayal Petroleum University, Raisan, Gandhinagar, India.

He has published 25 research papers in National/International peer reviewed journals. He has presented 22 research papers in various national and international conferences in India and abroad. Currently he is supervising Ph.D students. He is a life member of various national and international societies like ISTAM, CSI, ISTE, IANGE, CBEES, etc. 